\title{
Diachronic Tendency and Thinking Style Across the Broader Autism Spectrum: Sources of Individual Differences?
}

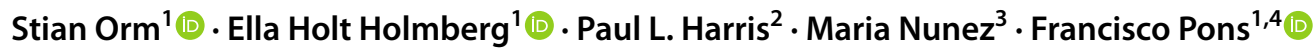

Accepted: 2 June 2021 / Published online: 7 July 2021

(c) The Author(s) 2021

\begin{abstract}
Objectives First, to see whether previous studies showing a limited capacity to spontaneously evoke the past and the future of a present moment (diachronic tendency) and a prevalence of mental images over inner speech (thinking style) in individuals with autism spectrum disorder could be replicated in individuals belonging to the broader autism phenotype. Second, to test the hypothesis that individuals thinking with mental images have a more limited diachronic tendency compared with individuals thinking with inner speech.

Methods Adults $\left(N=309, \mathrm{M}_{\text {age }}=31.5\right.$ years, $76 \%$ women $)$ with at least a high school degree were assessed with the Autism Spectrum Quotient, a test of diachronic tendency comprising four pictures varying in social interactivity and dynamicity, and a thinking style scale comprising three items representing three different everyday situations.

Results The results showed that adults with many autistic traits have a limited diachronic tendency but only when the situation is socially interactive and dynamic, think more in mental images than individuals with no or few autistic traits but nevertheless still think more with inner speech than with mental images, and the more the participants reported thinking in inner speech, the more they evoked past and future events when describing a socially interactive and dynamic situation. Conclusions More autistic traits are associated with a limited diachronic tendency in socially interactive and dynamic situations and more thinking in mental images, and thinking style could be one of the determinants of diachronic tendency in socially interactive and dynamic situations.
\end{abstract}

Keywords Temporal cognition $\cdot$ Diachronic tendency $\cdot$ Mental images $\cdot$ Inner speech $\cdot$ Autism spectrum disorders $\cdot$ Broader autism phenotype

Two separate conclusions have emerged over the last two decades among researchers and clinicians about the ways in which individuals with autism spectrum disorder (ASD) think about and apprehend time. First, a small number of studies using self-report measures about thinking style have shown that, unlike typically developing individuals, individuals with ASD are mainly visual as opposed to verbal

Francisco Pons

francisco.pons@psykologi.uio.no

University of Oslo, Oslo, Norway

Harvard University, Cambridge, MA, USA

3 Autonoma University of Madrid, Madrid, Spain

4 Faculty of Social Sciences, Department of Psychology, Section of Health, Developmental and Personality Psychology, University of Oslo, Blindern, P.O. Box 1094, NO-0317 Oslo, Norway thinkers (Dunne, 2008; Kunda \& Goel, 2011). Second, another recent line of research about diachronic tendency (i.e. the capacity to spontaneously evoke the past and the future of a present moment) has shown that individuals with ASD have difficulties in thinking spontaneously about events distributed across time. They have difficulties in evoking spontaneously the past and the future relative to the present moment (Boucher et al., 2007). These two assumptions are also supported by personal accounts of individuals with ASD (Grandin, 2009; Lawson, 2001; Williams, 1996).

An intriguing question is whether these two phenomena, thinking style and diachronic tendency, are related. If so, this could improve the understanding of ASD and pave the way for new research and interventions. Indeed, already in 2001, Boucher proposed a connection between these two phenomena. As Boucher (2001) pointed out, verbal thinking can make use of the time-based markers of language (i.e. on the temporal structure of language, on the use of 
tenses and temporal conjunctions) whereas visual thinking cannot (or less so). This difference between the verbal and the visual medium could help to explain differences between typical and non-typical individuals in their diachronic tendency. More specifically, because of its temporal markers, the verbal mode of thought could confer an advantage over the visual mode when individuals spontaneously consider the present moment in relation to the past and the future. No research has been conducted so far on the impact of thinking style on the diachronic tendency in typical and non-typical individuals. Only isolated case histories have been reported so far. For example, autistic individuals have noted that it is difficult for them to visualise the future when thinking in mental images (Grandin, 2009).

Two decades ago, the claim that individuals with ASD think mostly or only in mental images emerged (Harris \& Leevers, 2000). This claim has been empirically supported by several types of findings. First, studies have found that representing the mind by a picture-in-the-head or thoughtbubbles has a positive impact on the performance of individuals with ASD on false-belief tasks (McGregor et al., 1998a, b; Paynter \& Peterson, 2013; Swettenham, 1996; Wellman et al., 2002). Second, individuals with ASD showed better performance when presented with a picture-word pair than word-word pair on a semantic priming task, suggesting a visual thinking advantage (Kamio \& Toichi, 2000). This advantage has also been found in mental rotation tasks where some individuals with ASD showed enhanced abilities in forming, accessing, and manipulating mental images tasks (Soulières et al., 2011). Further, the performance of individuals with ASD on some cognitive tasks, like planning and taskswitching, have not been negatively affected by articulatory suppression to prevent the use of inner speech (Whitehouse et al., 2006; Williams et al., 2012, 2016). Lastly, individuals with ASD have reported using mostly, or only, mental images when asked about their thinking style (Dunne, 2008; Grandin, 2009; Hurlburt et al., 1994). However, replications are needed because studies have produced mixed results (see Williams et al., 2016 for a review) and some domains, e.g. self-report of thinking style, have rarely been researched.

Following the decline of the behaviorist dogma, introspection has again been considered a legitimate method for investigating people's inner experiences (Feest, 2012; Pons et al., 2012). Still, only two studies have investigated the thinking style of adults with ASD via self-report (Dunne, 2008; Hurlburt et al., 1994). Both studies found that adults with ASD reported thinking mostly or exclusively in mental images, whereas typically developing individuals and individuals with Specific Language Impairment (SLI) reported thinking mostly or exclusively in inner speech. These results are striking and warrant further investigation.

Around the same time as the claim about visual thinking in individuals with ASD emerged among researchers,
Boucher (2001) suggested that individuals with ASD had an impairment in temporal cognition. Since then, an increasing number of studies have investigated temporal cognition in individuals with ASD (see Allman \& Falter, 2015; Casassus et al., 2019; Jurek et al., 2019 for reviews). Some aspects, such as temporal thresholds and time sensitivity, have been extensively studied (Casassus et al., 2019). However, other aspects, such as the diachronic tendency, have been much less studied in individuals with ASD (Casassus et al., 2019). The diachronic tendency can be defined as the tendency to spontaneously evoke the past and the future relative to the present moment (e.g. Montangero, 1996; Montangero \& Pons, 1995). Numerous studies with typically developing individuals have shown that the diachronic tendency undergoes considerable change between middle childhood and adolescence independent of the gender of the individuals: 6-year-olds have the tendency to describe a situation as it is in the present moment (visible or invisible), 12-year-olds spontaneously refer to one or two past or future events, and adolescents and adults spontaneously evoke at least two past or future events. Research also showed that the diachronic tendency is related to social problem solving in typically developing children. For example, children with a good diachronic tendency are more capable of finding solutions to interpersonal conflicts (e.g. Montangero et al., 2000).

Only two experimental studies have investigated the diachronic tendency in individuals with ASD (Boucher et al., 2007). Boucher et al. found that children and adolescents with ASD scored significantly lower on diachronic tendency than controls with learning difficulties (the two groups were matched for age and intellectual capacities). When asked to describe pictures, individuals with ASD tended to spontaneously evoke events taking place in the present moment (visible or invisible) whereas individuals with learning difficulties spontaneously evoked past and future events as well.

As pointed out in recent reviews (Casassus et al., 2019; Jurek et al., 2019; Williams et al., 2016), the findings showing a restricted diachronic tendency and a visual thinking style in individuals with ASD need to be replicated. There is also a need to investigate the relation between these two phenomena in individuals with different levels of autistic traits. Among individuals with ASD, the prevalence of mental images over inner speech, could be one the reasons for their limited diachronic tendency, whereas among typical individuals, the prevalence of inner speech over mental images, could explain the facility with which they engage in diachronic tendency.

Over 20 years ago, Boucher (2001) noted that inner speech relies heavily on the time-based structure of language. Indeed, the sequence of words in language is temporally structured. Language also marks time in the form of tenses and in the standard narrative format, it involves a sequential recounting of events from the past to the present, 
and from the present to the future. In addition to its temporal structure and tenses, language also includes temporal conjunctions, nouns and adverbs (e.g. before, after, today, tomorrow, yesterday, and now). These various temporal features of language may help individuals who think mostly in inner speech to spontaneously consider the past and the future of a given moment. In contrast, mental images are by nature a less time-dependent system. Mental images can represent transformations over time, as in the classical image of a bridge depicting the passage from birth to death. A mental image can also be transformed over time as in mental rotation. However, a mental image can exist alone without necessarily being part of a series of images. Thus, the relative absence of temporal markers in mental images may be one of the reasons why individuals who think mostly in mental images do not think spontaneously about the past and the future of a given moment.

In summary, both inner speech and mental images can refer to time and to change over time (signified, referent). However, inner speech is fundamentally time-dependant whereas mental images are not (signifier). Inner speech cannot easily exist outside the dimension of time whereas mental images can. This difference could explain why individuals who think in inner speech are prone to reflect on the past and future of any given moment whereas individuals who think in mental images do not need to take the temporal dimension into consideration.

Two recent studies have suggested a link between language and temporal cognition in individuals with ASD. One study showed that children with ASD were less accurate in the interpretations of temporal conjunctions (Overweg et al., 2018); another study found that parents of children with ASD described their children as having difficulties with language about time (Poole et al., 2021). However, no research has been conducted so far on the impact of thinking style on the diachronic tendency, or the impact of autistic traits (rather than the ASD diagnosis) on the two phenomena.

The broader autism phenotype (BAP) refers to individuals with many autistic traits, but who may or may not meet the diagnostic criteria for ASD upon clinical assessment (Ingersoll \& Wainer, 2014). In recent years, there has been an increasing interest in autism symptomology as a spectrum across the population, ranging from those with no autistic traits to those with many. Autistic traits in the general population, like the ASD diagnosis, have been found to be under genetic influence (Hoekstra et al., 2007; Nayar et al., 2021). Within the general population, more autistic traits have been found to be associated with poorer interpersonal functioning, more psychological distress, and feelings of loneliness (Jobe \& Williams White, 2007; Lundin et al., 2019). The most frequently used tool to assess the BAP has been the Autism Spectrum Quotient (AQ; Baron-Cohen et al., 2001; Ingersoll $\&$ Wainer, 2014). In the current study, the 10 -item short version of the AQ, the AQ-10 is used (Allison et al., 2012). The AQ- 10 has been shown to perform in a comparable fashion to the original version of 50 items, and has been found to be a suitable tool to assess autistic traits across the spectrum (Booth et al., 2013; Lundin et al., 2019). No previous study has investigated how thinking style and diachronic tendency vary both as a result of autistic traits across the spectrum and in terms of categorical divisions between individuals belonging to the BPA and individuals with no or few autistic traits. Taking advantage of this combined approach to ASD as both a dimension and discrete categories can provide important knowledge about how ASD affects peoples thinking style and temporal cognition (Happé \& Frith, 2020a, b).

The hypothesis in this study is that the temporal dynamicity in language makes it easier to represent changes over time, to evoke the past and the future of a present moment, with inner speech than mental images (Pons et al., 2012). Thus, limited inner speech or an over-reliance on mental images should restrict the diachronic tendency. Given that both the diachronic tendency and thinking style may be either impaired or biased in individuals with ASD, this study investigates their relation in individuals with different levels of autistic traits. It is hypothesised that individuals with a higher number of autistic traits, i.e. individuals belonging to the BAP, will display a limited diachronic tendency and think more in mental images than individuals with none or fewer autistic traits.

The current study had two main goals: first, to see whether the few previous studies showing a limited diachronic tendency (i.e. a limited spontaneous temporal perspective taking) and a biased thinking style (i.e. a dominance of mental images over inner speech) in individuals with ASD could be replicated as a function of the number of autistic traits. This prediction was tested by examining how diachronic tendency and thinking style are linked to the number of autistic traits measured by the AQ-10. Second, to test the hypothesis that individuals who think primarily with mental images have more difficulties in spontaneously evoking the past and the future of a present moment than individuals who think with inner speech.

\section{Method}

\section{Participants}

Three-hundred-and-thirty-two adults with varying levels of autistic traits were tested. Of these, 23 were excluded because they did not have a high school degree and we wanted a relatively homogenous sample in terms of level of education so that differences in the participants' performances could not be attributed to differences in their overall intellectual level. The final sample comprised 
309 participants between the age of 18 and 40 years ( $M=31.5$ years, $\mathrm{SD}=6.2$ years). Three-quarters were women ( $76 \%$ women, $23 \%$ men, $1 \%$ other).

\section{Procedures}

Participants were recruited through acquaintances, social media, and lectures at the University of Oslo, and through events and online groups organised by the Norwegian Autistic Society (Autismeforeningen i Norge) and the Norwegian autistic self-advocacy organisation (Spekteret). We used this broad recruitment strategy to obtain a large sample of participants with different levels of autistic traits. Despite the advantages of this inclusive recruitment approach, it is offset by certain limitations.

Completing the questionnaire was voluntary and anonymous. The study was prospectively reviewed and approved by the ethical review board at the institution where the work was conducted. Data were collected online and took approximately $12 \mathrm{~min}(\mathrm{SD}=8 \mathrm{~min})$ to complete. The questionnaire included an informed consent form, background questions (age, gender, and education), assessment of diachronic tendency, thinking style questions, and the Autism Spectrum Quotient short version (AQ-10) for adults.

\section{Measures}

The participants' diachronic tendency was assessed with four pictures (see Fig. 1). Three of them (1,2 and 4) were similar to those used in previous studies with typically and non-typically developing children, adolescents and adults (e.g. Boucher et al., 2007; Montangero, 1996; Montangero \& Pons, 1995; Montangero et al., 2000; Moore et al., 2014; Pons et al., 2002). However, all pictures were redrawn by the same artist, to forestall the possibility that participants would pay attention to different drawing styles in their responses. For each picture, participants were first asked to: "Describe what is going on in this picture" and then prompted to expand on their responses: "Could you say something else about this picture". Participants were asked to write down their answers (whereas in previous studies they just had to say them aloud). The presentation of the four pictures was partially counterbalanced (i.e. participants received the pictures in four different, randomised orders). The pictures were drawn with different levels of social interactivity and dynamism. Pictures 1 and 4 were dynamic (i.e. ongoing physical action) whereas Pictures 3 and 4 were social interactive (i.e. ongoing social interaction). Thus, one picture was dynamic but not socially interactive (picture 1), one was static and not socially interactive (picture 2), one was socially interactive but static (picture 3 ), and one was socially interactive and
Fig. 1 Pictures used to assess the diachronic tendency

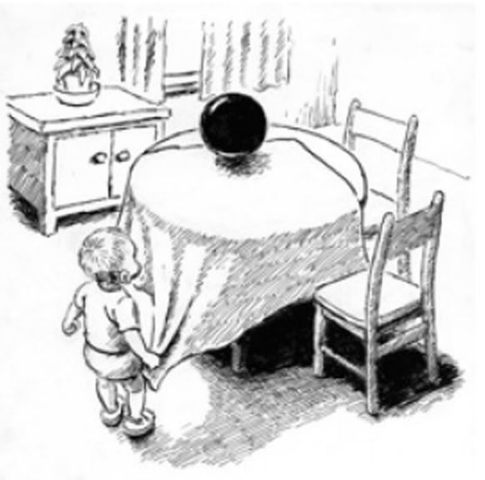

Picture 1. Non-social and dynamic picture

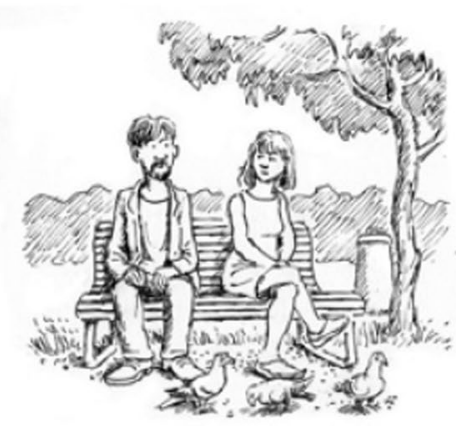

Picture 3. Social and non-dynamic picture

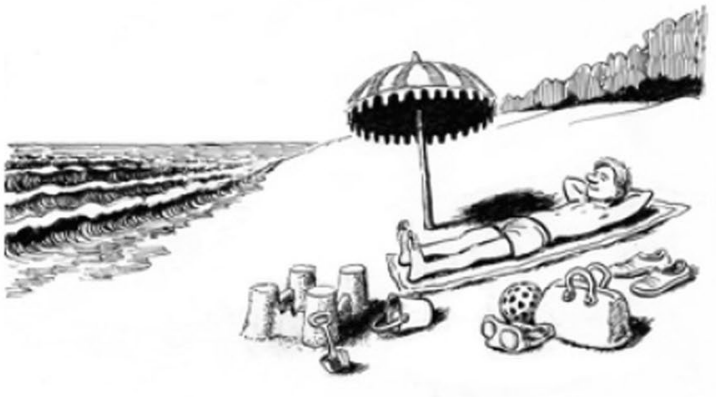

Picture 2. Non-social and non-dynamic picture

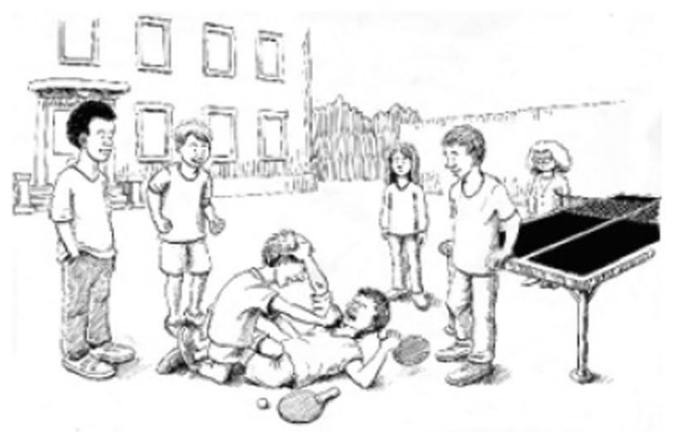

Picture 4. Social and dynamic picture 
dynamic (picture 4) (see Fig. 1). Participants' descriptions of each picture were scored for the number of diachronic events spontaneously evoked in the description. We used the same scoring criteria as earlier studies (e.g. Boucher et al., 2007; Montangero \& Pons, 1995). An event was considered to be diachronic when it referred to the past or future of the situation represented in the picture, independent of the tense of the verb used to describe the event or its plausibility. Some examples of diachronic responses were: "The ball is going to roll down (future event) and possibly hit the child" (future event), "Something is about to happen" (future event) (picture 1); "The man has built a sandcastle (past event) and a boat has passed by and created the waves (past event)", "The man walks on the beach (past event)" (picture 2); "The man has fed the pigeons (past event) and they have had a fight (past event)", "A disaster is waiting" (future) (picture 3); "They play ping pong (past event) and the teacher is coming to stop the fight (future event)"(picture 4). Two raters read the participants responses independently and inter-rater reliability based on more than one third of randomly selected participants (37\%) was good (intra-class correlation [ICC]; two-way random $=0.72-0.83, p<0.000)$. Analyses showed that the number of diachronic responses reported by the participants' was not internally consistent $(\alpha=0.56)$ and significantly different $(F(3,924)=90.44, p<0.000)$ across the four pictures (Table 1). Accordingly, we decided to retain participants' scores on each of the four diachronic tendency questions as measures of their diachronic tendency.

The method used to assess thinking style was based on the work of Dunne (2008), but the response scale was changed from open-ended to a Likert-scale to facilitate the data collection and analyses. The participants were asked to say whether they think in inner speech or mental images on a four-point Likert scale ranging from "Only mental images" (0), "Mostly mental images" (1), "Mostly inner speech" (2) to "Only inner speech" (3) in three different everyday situations: (i) when they were thinking about their shopping list, (ii) when they were thinking in general, and (iii) when they were thinking about performing an action. Analyses showed that the three thinking style questions were internally consistent $(\alpha=0.75)$ and that there were no significant differences between the participants' scores on the different thinking style questions (Table 2). Accordingly, we decided to use the participants' average score on the three thinking style questions as a measure of their thinking style.

We used the Autism Spectrum Quotient 10-items (AQ10) for adults (Allison et al., 2012) to assess the participants' number of autistic traits. AQ-10 has been shown to have excellent validity and a positive predictive value of 0.85 for the Autism Spectrum Diagnosis (Allison et al., 2012). Good sensitivity (0.80-0.88) and specificity $(0.87-0.91)$ have been reported in samples of individuals with ASD and TD individuals (Allison et al., 2012; Booth et al., 2013). Analyses showed that AQ-10 was internally consistent $(\alpha=0.76)$. A
Table 1 Diachronic tendency: number of past and future events evoked spontaneously by picture for the total sample and by extreme group (BAP and few autistic traits)

Table 2 Thinking style by picture for the total sample and by extreme group (BAP and few autistic traits)

\begin{tabular}{|c|c|c|c|c|c|c|}
\hline \multirow[t]{2}{*}{ Figure } & \multicolumn{2}{|c|}{$\begin{array}{l}\text { Total sample } \\
(N=309)\end{array}$} & \multicolumn{2}{|c|}{$\begin{array}{l}\text { Broader autism phe- } \\
\text { notype }(N=43)\end{array}$} & \multicolumn{2}{|c|}{$\begin{array}{l}\text { Few autistic } \\
\text { traits }(N=55)\end{array}$} \\
\hline & $M$ & SD & $M$ & SD & $M$ & SD \\
\hline Picture 1 (non-social/dynamic) & 0.98 & 0.64 & 1.06 & 0.77 & 0.95 & 0.59 \\
\hline Picture 2 (non-social/non-dynamic) & 0.85 & 0.97 & 0.82 & 1.07 & 0.95 & 0.83 \\
\hline Picture 3 (social/non-dynamic) & 0.22 & 0.48 & 0.20 & 0.41 & 0.24 & 0.47 \\
\hline Picture 4 (social/dynamic) & 0.61 & 0.70 & 0.49 & 0.65 & 0.82 & 0.70 \\
\hline
\end{tabular}

Broader autism phenotype $=$ individuals scoring $\geq 8$ on the AQ-10 (BAP), few autistic traits =individuals scoring $\leq 1$ on the AQ-10. Levene's test of equality of variances suggested equality of variances in the broader autism phenotype and the few autistic traits groups

\begin{tabular}{|c|c|c|c|c|c|c|}
\hline \multirow[t]{2}{*}{ Question } & \multicolumn{2}{|c|}{ Total sample $(N=309)$} & \multicolumn{2}{|c|}{$\begin{array}{l}\text { Broader autism phenotype } \\
(N=43)\end{array}$} & \multicolumn{2}{|c|}{$\begin{array}{l}\text { Few autistic traits } \\
(N=55)\end{array}$} \\
\hline & $M$ & SD & $M$ & SD & $M$ & SD \\
\hline Question 1 (shopping) & 1.80 & 0.77 & 1.71 & 0.89 & 1.91 & 0.73 \\
\hline Question 2 (thinking) & 1.74 & 0.58 & 1.63 & 0.60 & 1.87 & 0.58 \\
\hline Question 3 (doing) & 1.75 & 0.62 & 1.51 & 0.71 & 1.82 & 0.58 \\
\hline
\end{tabular}

$0=$ only mental image, $1=$ mainly mental image, $2=$ mainly inner speech, $3=$ only inner speech

Broader autism phenotype $=$ individuals scoring $\geq 8$ on the AQ-10 (BAP), few autistic traits =individuals scoring $\leq 1$ on the AQ-10 
one-sample $t$-test showed that our sample had on average a significantly $(t(308)=9.92, p<0.000)$ higher number of autistic $(M=3.9$ traits, $\mathrm{SD}=2.7)$ traits than a comparable Swedish general population sample $(M=2.4$ traits, $\mathrm{SD}=3.0)$ (Lundin et al., 2019). This is likely to be because many participants in our study were approached through two associations for individuals with ASD.

\section{Community Involvement}

In line with the recent emphasis on including adults with ASD in the research process (e.g. Jivraj et al., 2014; Nicolaidis et al., 2019), the research team included an autistic researcher. More specifically, the idea for this study was a result of conversations between the researcher with ASD and colleagues in the research team. Thus, the researcher with ASD was central to the development of the study and was involved in all its stages from designing and executing the study to the data analyses and interpretation of the results.

\section{Data Analyses}

Statistical analyses were conducted with IBM SPSS Statistics version 25 . The results are presented in two parts. First, we analysed the impact of autistic traits on participants' diachronic tendency and thinking style to check whether there is a dimensional relationship between these two phenomena, across the spectrum of autistic traits. In addition, to examine the categorical impact of autistic traits on diachronic tendency and thinking style, analyses were conducted with two extreme groups representing almost one third (32\%, $N=98)$ of the entire sample: Participants scoring low on the AQ-10 (zero or one autistic trait, $M=0.75$ trait, $\mathrm{SD}=0.44$, $N=55$ ) and participants representing the BAP, scoring high on the AQ-10 (eight autistic traits or more, $M=9.00$ traits, $\mathrm{SD}=0.87, N=43$ ). Mean age was similar in these two extreme groups (respectively, $M=31.8$ years $(\mathrm{SD}=6.5$ ) and 30.8 years $(\mathrm{SD}=6.0)$ ) whereas gender was significantly different $\left(\mathrm{chi}^{2}(2)=11.47, p<0.01\right)$. More than two-thirds of the men (70\%) scored at the BAP level whereas only one third of the women (34\%) did. This difference is in accordance with the literature; autistic traits are more prevalent among men than women (Loomes et al., 2017).

Second, we analysed the impact of thinking style on diachronic tendency to test the hypothesis that individuals who think more in terms of mental images have greater difficulties in evoking the past and future of a present moment than individuals who think more in terms of inner speech. An analysis of the impact of thinking style on the diachronic tendency was also conducted with two extreme groups of participants representing more than two-thirds $(69 \%$, $N=213$ ) of the total sample: participants thinking only or mainly in mental images (i.e. scoring only 0 or $1, N=46$ ) and participants thinking only or mainly in inner speech (scoring only 2 or $3, N=167$ ) across all three thinking style questions. Mean age and gender were similar in these two groups (mental image prevalence group: $M=31.6$ years $(\mathrm{SD}=6.4)$ and $78 \%$ of women; inner speech prevalence group: 32.3 years (5.9) and $79 \%$ of women).

\section{Results}

\section{Impact of Autistic Traits on Diachronic Tendency}

One-sample $t$-test analyses showed that, for all four pictures, the participants with a low number of autistic traits reported significantly fewer past and future events (see Table 1) than typically developing adolescents and adults tested in previous studies (i.e. an average of two diachronic events) (e.g. Montangero \& Pons, 1995; Montangero et al., 2000): picture $1(t(54)=-13.24, p<0.000)$, picture $2(t(54)=-9.47$, $p<0.000)$, picture $3(t(54)=-27.83, p<0.000)$ and picture $4(t(54)=-12.59, p<0.000)$.

Correlational analysis with the entire sample showed no significant association between the number of autistic traits reported by the participants and the number of diachronic events they evoked for the four pictures. A regression analysis with the entire sample in which the diachronic tendencies on pictures 1, 2, 3 and 4 were the dependent variables and age, gender and the number of autistic traits were the independent variables showed no significant association between these four variables and no significant effect of age, gender or the number of autistic traits. There was no significant association between participant's number of autistic traits and the length of their responses to the pictures used to assess diachronic tendency.

An examination of Table 1 showed that for three of the four pictures (2, 3 and 4), participants with a high number of autistic traits tended to evoke fewer past and future events than participants with a low number of autistic traits. However, these differences were significant only for picture 4 $(t(96)=2.37, p<0.05)$; participants without autistic traits reported almost double the number of past and future diachronic events than participants belonging to the BAP, when asked to describe the socially interactive and dynamic situation depicted in picture 4 (see Fig. 2).

A chi-square analysis revealed a significant difference in the number of participants belonging and not belonging to the BAP evoking or not evoking past or future events to describe picture 4 (chi-square $(1, N=98)=8.56, p<0.005$ ). The majority of the participants describing the picture by referring only to the present (i.e. with no reference to past or future events) had belonged to the $\operatorname{BAP}(61 \%$; AQ $\geq 8)$ whereas the majority evoking at least one (and up to four) 
Fig. 2 Diachronic tendency score at picture 4 in the two extreme groups (BAP and few autistic traits). Note. Few autistic traits group $(N=55)=$ AQ-10 score $\leq 1$; BAP group $(N=43)=$ AQ-10 score $\geq 8$

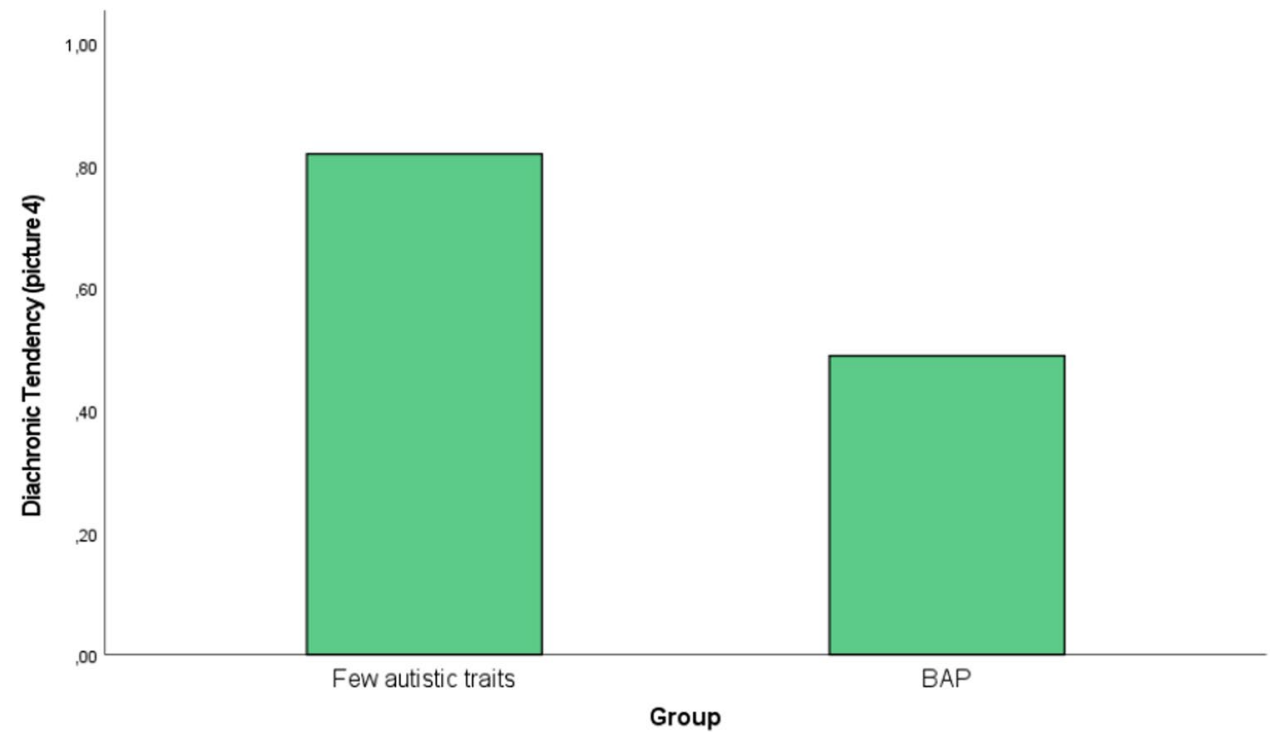

past or future events to describe this picture had few autistic traits (69\%; i.e. did not belong to the BAP; $A Q \leq 1)$.

\section{Impact of Autistic Traits on Thinking Style}

Correlation analysis with the entire sample showed a significant relation between the number of autistic traits reported by participants and their propensity to think in mental images rather than inner speech $(r(307)=-0.11$, $p<0.05$ ); the higher the number of reported autistic traits, the less they reported thinking in inner speech. A regression analysis with the entire sample where thinking style was the dependent variable and age, gender and the number of autistic traits were the independent variables showed no significant association between these four variables, no significant effect of age and gender but a significant effect for the number of autistic traits (beta $=-0.10, t=-1.68$, $p<0.05$ (one-tailed), partial $r=-0.10$ ).

Although participants with a high number of autistic traits reported thinking more with inner speech than with mental images $(M=1.6, \mathrm{SD}=0.6)$, they still reported thinking with inner speech significantly less $(t(96)=1.76$, $p<0.05$ (one-tailed)) than participants with a low number of autistic traits $(M=1.9, \mathrm{SD}=0.5)$ (see Fig. 3).

A chi-square analysis revealed a significant difference in the number of participants belonging and not belonging to the BAP who reported thinking with mental images or inner speech (chi-square $(1, N=72)=4.35, p<0.05)$ : The majority of participants thinking with mental images belonged to the BAP $(69 \%)$ whereas the majority of

Fig. 3 Overall thinking style score in the two extreme groups (BAP and few autistic traits). Note. Few autistic traits group $(N=55)=$ AQ-10 score $\leq 1$; BAP group $(N=43)=\mathrm{AQ}-10$ score $\geq 8$. Overall thinking style: $0=$ only mental image, $1=$ mainly mental image, $2=$ mainly inner speech, $3=$ only inner speech

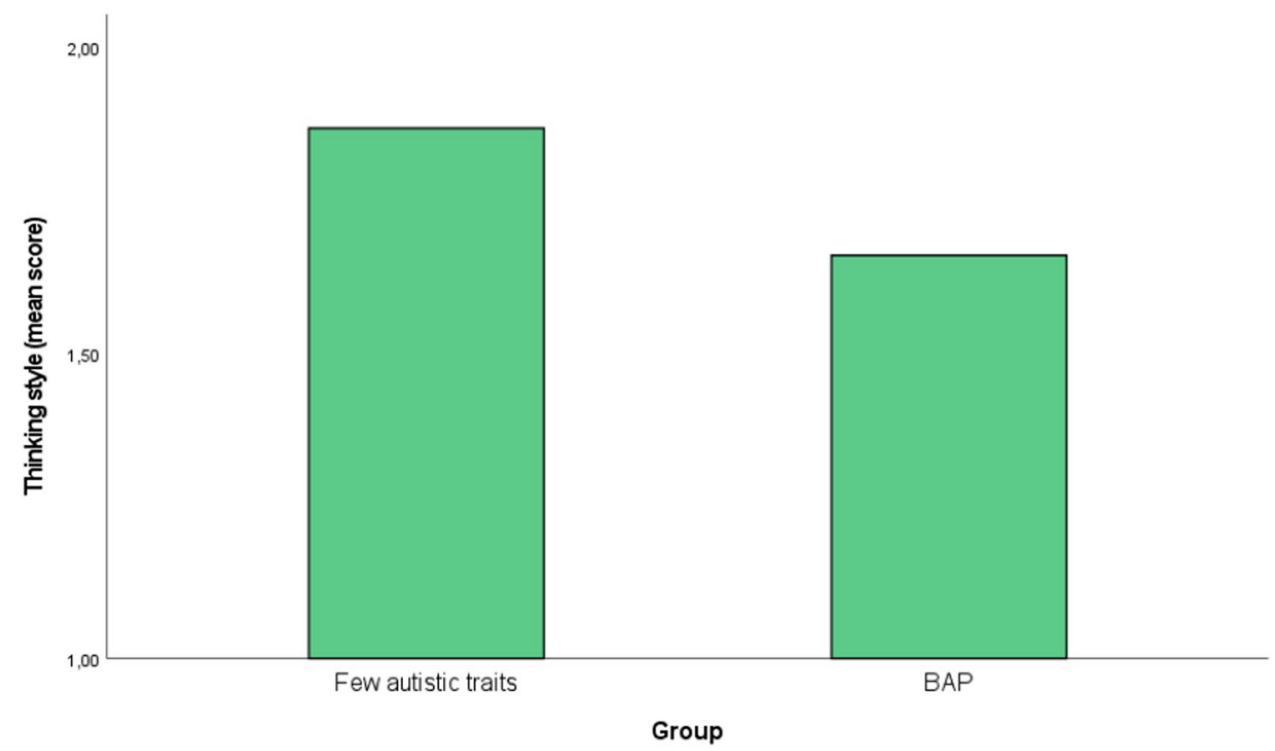


participants thinking with inner speech did not belong to the BAP $(61 \%)$.

\section{The Impact of Thinking Style on the Diachronic Tendency}

Correlational analyses based on the entire sample revealed only one significant (one-tailed) positive correlation ( $r$ $(307)=0.10, p<0.05)$ between thinking style and the diachronic tendency (i.e. for picture 4); the more the participants reported thinking in inner speech the more they evoked past and future events when describing a socially interactive and dynamic situation. This relationship was not affected by the number of autistic traits (partial $r(306)=0.10, p<0.05$ ). A regression analysis in which the diachronic tendency for the socially interactive and dynamic situation (picture 4) was the dependent variable and age, gender and thinking style were the independent variables showed no significant association between these four variables, no significant effect of gender and age, but a significant (one-tailed) effect of thinking style on diachronic tendency (beta $=0.11, t=1.88$, $p<0.05$, partial $r=0.11$ ). There was no significant association between participants thinking style and the length of their responses to the pictures used to assess diachronic tendency.

Analyses with the participants reporting a clear propensity to think either in mental image or inner speech confirmed the previous analyses. Participants thinking in inner speech evoked significantly $(t(211)=2.44, p<0.05)$ more past and future events $(M=0.7, \mathrm{SD}=0.8)$ than participants thinking in mental images $(M=0.4, \mathrm{SD}=0.6)$; they evoked almost twice as many diachronic events when they were asked to describe a socially interactive and dynamic situation (picture 4) (see Fig. 4). This difference also remained when excluding the participants belonging to the BAP ( $t$ $(178)=2.81, p<0.01)$.

A chi-square analysis revealed a significant difference in the number of participants thinking in mental images or inner speech who did or did not evoke past or future events to describe picture 4 (chi-square $(1, N=213)=5.95, p<0.05)$ : The majority of participants thinking with mental images described the picture by referring only to the present $(65 \%)$ whereas the majority thinking with inner speech evoked at least one (and up to four) past or future events (55\%).

\section{Discussion}

The study had two objectives: first, to see whether the few previous studies showing limited diachronic tendency (i.e. difficulties in spontaneously evoking the past or future of a present moment) and a biased thinking style (i.e. thinking with mental images rather inner speech) in individuals with ASD could be replicated in a sample of individuals belonging to the BAP and across the spectrum depending on the degree of autistic traits. Second, to test the hypothesis that individuals thinking with mental images have more difficulties in spontaneously evoking the past and the future of a present moment as compared to individuals thinking with inner speech.

The results partially replicated the results obtained in previous studies with respect to the diachronic tendency in children and adolescents with ASD (Boucher et al., 2007). They showed that adults with many autistic traits have a limited diachronic tendency but only when the situation is socially interactive and dynamic (i.e. picture 4). This finding can be interpreted in different ways. Why the adults with and without ASD had similar performances on the diachronic
Fig. 4 Diachronic tendency score at picture 4 (socially interactive and dynamic) by overall thinking style extreme group (Mental Image and Inner Speech). Note. Mental images extreme group $(N=46)=$ participants thinking only or mainly in mental images (i.e. scoring only 0 or 1 on the thinking style questionnaire); inner speech extreme group $(N=167)=$ participants thinking only or mainly in inner speech (scoring only 2 or 3 on the thinking style questionnaire)

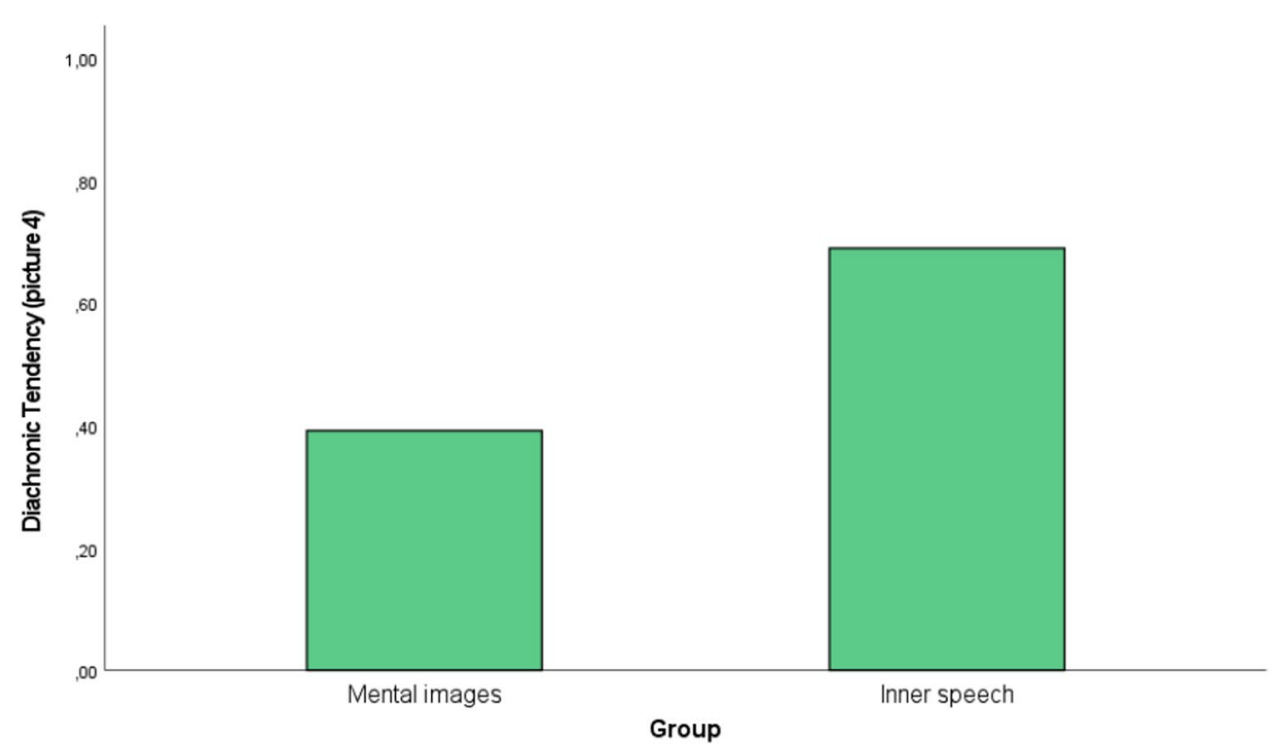


tendency for most situations (three of the four pictures)? Note that in the current study, the method used to assess the diachronic tendency was somewhat different from the method used in previous studies; i.e., in this study, participants were asked to write down their answers whereas in previous studies, they only had to say them aloud (e.g. Boucher et al., 2007; Montangero \& Pons, 1995; Montangero et al., 2000). This difference in method may have led to a floor effect. Indeed, the adults with few autistic traits in this study displayed a lower level of diachronic responding than typically developing adolescents and adults in previous studies, irrespective of the picture they were asked to describe. Such a floor effect could explain why no difference was between individuals with many and few autistic traits; the diachronic tasks were too demanding. Further, the method can have been beneficial for adults with many autistic traits. Crane et al. (2013) argued that writing responses rather than verbalising them to an examiner can reduce social anxiety in individuals with ASD, and thereby enhance their performance. It would be interesting in the future to figure out which interpretation is better by comparing within the same individuals these different methods (i.e. writing down their answer on a computer versus giving their answer orally to an examiner).

Another interpretation of the limited impact of autistic traits on the diachronic tendency could be that adults belonging to the BAP actually have an intact diachronic tendency. This interpretation is in accordance with the differential developmental trajectory hypothesis proposed by Casassus et al. (2019): Children and adolescents with ASD have a limited diachronic tendency (Boucher et al., 2007) but they catch up during adulthood. It would be interesting in the future to test Casassus' hypothesis by using the same methodology to test children, adolescents, and adults with and without ASD to see whether age impacts the differential diachronic tendency of individuals with few versus many autistic traits (i.e. the older, the smaller the difference).

How to interpret the fact that in one of the situations, the socially interactive and dynamic one (i.e. picture 4), the adults with many autistic traits displayed a limited diachronic tendency? One possible answer is that children with many autistic traits orient themselves more towards the nonsocial than the social environment (e.g. Gale et al., 2019). Thus, by adulthood, individuals belonging to the BAP have developed adequate temporal concepts for non-socially interactive events. An early-onset impairment in orientation to social stimuli might have cascading effects on deficits in social cognition, and these deficits could prevent individuals with many autistic traits from developing adequate temporal concepts of socially interactive events, at least when these events reach the complexity of the social interaction depicted in picture 4 . The level of interactivity and hence the degree of interpretation may be central to understanding the group difference on picture 4; the more complex social interaction a picture depicts, the larger the group differences between individuals with few or many autistic traits are. This interpretation is in line with the findings of a recent meta-analysis showing that adults with ASD have a broad impairment in the social cognitive domain but not in the non-social cognitive domain (Velikonja et al., 2019).

To understand complex socially interactive situations and predict future outcomes, the ability to adopt different temporal perspectives towards these situations is necessary. If individuals with many autistic traits do not develop temporal concepts of complex socially interactive situations, this could affect their social competence (e.g. Boucher, 2001), and should be considered a target for social skills intervention. It would be interesting to test the difference between socially interactive and non-socially interactive diachronic tendency in the future by re-running a developmental study (longitudinal rather than cross-sectional) with children, adolescents and adults with and without ASD and to assess them for their diachronic tendency in socially interactive and non-socially interactive situations. Finally, it should be noted that the results fully replicated those obtained in previous studies showing that gender does not impact the diachronic tendency in children, adolescents and adults with few or many autistic traits (e.g. Boucher et al., 2007; Montangero \& Pons, 1995; Montangero et al., 2000). Whatever their age or typicality, gender does not impact spontaneous temporal perspective-taking.

The results only partially replicated the results obtained in previous studies regarding the thinking style of individuals with ASD (Dunne, 2008; Hurlburt et al., 1994). Indeed, the results confirmed that individuals belonging to the BAP think less in inner speech than individuals with few autistic traits. Nevertheless, they still think more with inner speech than with mental images. By contrast, previous studies showed that individuals with ASD think more with mental images than inner speech. At least two methodological reasons could explain this discrepancy. First, in previous studies the participants were asked to report in the context of an open interview whether they were thinking with mental images and with inner speech independently whereas in this study these two dimensions were opposed to each other in one single Likert scale. Second, as pointed by Williams et al. (2016), most of the studies on verbal thinking and inner speech in individuals with ASD, like those of Dunne (2008) or Hurlburt et al. (1994), mainly rely on verbal presentations (versus pictorial presentations) and on verbal report (versus visual scales). This methodological difference makes the interpretation of any differences in thinking style between individual with and with ASD problematic because many individuals with ASD have more or less severe language impairments (syntactic, sematic, pragmatic) (Boucher, 2012; Eigsti et al., 2011; Kwok et al., 2015). Are these differences 
in thinking style related to the way it is measured (via almost exclusively verbal descriptions and questions rather than via pictures and images) or are these differences in thinking style genuinely related to the way that participants are thinking (inner speech versus mental images)? Indeed, as mentioned previously, some research on Theory of Mind in individuals with ASD has shown that representing the mind by a picture-in-the-head, photo-in-the-head, or thought-bubbles has a positive impact on their performance in false-belief tasks (McGregor et al., 1998a, b; Paynter \& Peterson, 2013; Swettenham, 1996; Wellman et al., 2002). In summary, it is too early to conclude whether individuals with many autistic traits have a limited or a different thinking style compared to individuals without ASD.

In the future, it would be interesting to assess independently with two different Likert scales the use of inner speech and mental images, using verbal and non-verbal descriptions and questions about everyday situations, to check whether many autistic traits reverses people's thinking style from inner speech to mental images (in line with the results of Dunne, 2008 and Hurlburt et al., 1994) or simply attenuates their propensity to think with inner speech (in line with the results of this study). Such a study would also help to answer the question of whether the thinking style of individuals with ASD is the result of a limitation in language (more or less compensated by mental images) and/or to an overdeveloped capacity for mental images (and more or less intact inner speech).

The second objective of this study was to test the hypothesis that it is more difficult to represent changes over time and to spontaneously evoke the past and the future of a present moment with mental images than with inner speech, given the more dynamic nature of speech compared to images; a limitation in inner speech or an over-reliance on mental images should lead to a limited diachronic tendency (Pons et al., 2012). The results showed some promising findings that warrant further investigation. The more participants reported thinking in inner speech, the more they evoked past and future events when describing a socially interactive and dynamic situation (i.e. picture 4). Individuals thinking in inner speech evoked almost twice as many past and future events as compared to those thinking in mental images (autistic traits did not affect this relation). This result can be interpreted in different ways. The impact of thinking style on the diachronic tendency is the exception whereas the no relationship is the norm. Indeed, this relationship was found only once (out of four different situations). This result confirms the results from earlier studies showing that the diachronic tendency is a rather specific and distinct cognitive ability (e.g. Boucher et al., 2007; Pons \& Montangero, 1999). However, previous studies have also showed that the diachronic tendency is related to social-problem solving in typically developing children (e.g. Montangero, 1996;
Montangero et al., 2000). This study, together with these earlier studies, suggests that there is a privileged relationship between the diachronic tendency and social and non-social problem-solving in individuals with few and many autistic traits. It would be interesting in the future to investigate systematically the impact of thinking style on the diachronic tendency in socially interactive and less socially interactive situations.

\section{Limitations and Future Research Directions}

Two limitations regarding the generalizability of the sample should be noted. First, the inclusion criteria, "having at least a high-school degree", imply that the sample is cognitively high-functioning, and this factor could have impacted findings. The level of cognitive functioning might explain the lack of larger group differences (e.g. Boucher, 2001; Gil et al., 2012; Vogel et al., 2019). In the future, assessments of general cognitive and linguistic abilities should be included in order to account for individual differences in these domains. Second, despite the AQ being one of the most common measures to assess autistic traits across the population, the ability of the AQ to discriminate between ASD and other mental health disorders is uncertain (Wigham et al., 2019). Thus, given the inclusive approach to recruitment, some of the participants may have obtained a high score on the AQ for reasons other than having many autistic traits (e.g. due to other mental health disorder or general sociocognitive difficulties). However, this inclusive strategy to recruitment is consistent with a recent, increasingly powerful approach in clinical research that supports dimensional rather than categorical distinctions in approaching mental health disorders (RDoC; Cuthbert, 2015).

In further research, it would also be interesting to include a question about how participants are "thinking about time". Such a question might provide additional information about how people with different levels of autistic traits think about time, and how their thinking about time relates to their diachronic tendency. It would also be interesting to explore the thinking style and diachronic tendency of typical adults in relation to their profession, giving the opportunity to assess (i) whether, for example, professional artists (painters, designers) think more in mental imagery and less in inner speech as compared to professional writers (fictional and non-fictional) and (ii) whether thinking style is one of the factors that impacts the diachronic tendency in typically developing professional adults.

The findings from this study seen in connection with the result of Boucher et al. (2007) suggests that the limitations in diachronic tendency may be more pronounced in children and adolescents as opposed to adults, and in individuals with lower cognitive and linguistic abilities as opposed to "highfunctioning" individuals. Thus, further research should test 
the hypotheses that the limitations in diachronic tendency associated with autistic traits attenuate with age and cognitive and linguistic abilities. Further, longitudinal research is also needed to investigate the developmental trajectories of diachronic tendency in individuals across the autism spectrum, and should also investigate the hypothesis that the diachronic tendency of non-socially interactive versus socially interactive situations follows different developmental trajectories.

Acknowledgements We would like to thank all the participants for taking part in this study and Mikael Homberg for drawing the pictures.

Author Contribution SO designed and executed the study, analysed the data, and wrote and edited the manuscript. EHH designed and executed the study, analysed the data, and wrote and edited the manuscript. PLH collaborated with data analyses, interpreted results, and wrote and edited the manuscript. MN collaborated with data analyses, interpreted results, and wrote and edited the manuscript. FP designed and executed the study, analysed the data, and wrote and edited the manuscript.

Funding Open access funding provided by University of Oslo (incl Oslo University Hospital). MN was funded by the Spanish Ministry of Science and Innovation (BGP18/00186) and the Madrid Regional Government (S12/PBG/2020-00016).

\section{Declarations}

Ethics Approval This study was prospectively reviewed and approved by the Ethical Board of the Department of Psychology at the University of Oslo (reference number: 3700686). All participants provided informed consent prior to participating in the study.

Conflict of Interest The authors declare no competing interests.

Open Access This article is licensed under a Creative Commons Attribution 4.0 International License, which permits use, sharing, adaptation, distribution and reproduction in any medium or format, as long as you give appropriate credit to the original author(s) and the source, provide a link to the Creative Commons licence, and indicate if changes were made. The images or other third party material in this article are included in the article's Creative Commons licence, unless indicated otherwise in a credit line to the material. If material is not included in the article's Creative Commons licence and your intended use is not permitted by statutory regulation or exceeds the permitted use, you will need to obtain permission directly from the copyright holder. To view a copy of this licence, visit http://creativecommons.org/licenses/by/4.0/.

\section{References}

Allison, C., Auyeung, B., \& Baron-Cohen, S. (2012). Toward brief "red flags" for autism screening: The Short Autism Spectrum Quotient and the Short Quantitative Checklist in 1,000 cases and 3,000 controls. Journal of the American Academy of Child \& Adolescent Psychiatry, 51(2), 202-212.e7. https://doi.org/10.1016/j.jaac.2011.11.003

Allman, M. J., \& Falter, C. M. (2015). Abnormal timing and time perception in autism spectrum disorder? A review of the evidence. In A. Vatakis \& M. J. Allman (Eds.), Time distortions in mind - temporal processing in clinical populations (pp. 39-56). Brill Open.
Baron-Cohen, S., Wheelwright, S., Skinner, R., Martin, J., \& Clubley, E. (2001). The Autism-Spectrum Quotient (AQ): Evidence from Asperger syndrome/high-functioning autism, males and females, scientists and mathematicians. Journal of Autism and Developmental Disorders, 31(1), 5-17. https://doi.org/10.1023/A:10056 53411471

Booth, T., Murray, A. L., McKenzie, K., Kuenssberg, R., O’Donnell, M., \& Burnett, H. (2013). Brief report: An evaluation of the AQ-10 as a brief screening instrument for ASD in adults. Journal of Autism and Developmental Disorders, 43(12), 2997-3000. https://doi.org/10.1007/s10803-013-1844-5

Boucher, J. (2001). Time-parsing and autism. In C. Hoerl \& T. McCormack (Eds.), Time and memory: Issues in philosophy and psychology (pp. 111-135). Clarendon Press.

Boucher, J. (2012). Research review: Structural language in autistic spectrum disorder-Characteristics and causes. Journal of Child Psychology and Psychiatry, 53(3), 219-233. https://doi.org/10. 1111/j.1469-7610.2011.02508.x

Boucher, J., Pons, F., Lind, S., \& Williams, D. (2007). Temporal cognition in children with autistic spectrum disorders: Tests of diachronic thinking. Journal of Autism and Developmental Disorders, 37(8), 1413-1429. https://doi.org/10.1007/ s10803-006-0285-9

Casassus, M., Poliakoff, E., Gowen, E., Poole, D., \& Jones, L. A. (2019). Time perception and autistic spectrum condition: A systematic review. Autism Research, 12(10), 1440-1462. https://doi. org/10.1002/aur.2170

Crane, L., Lind, S. E., \& Bowler, D. M. (2013). Remembering the past and imagining the future in autism spectrum disorder. Memory, 21(2), 157-166. https://doi.org/10.1080/09658211.2012.712976

Cuthbert, B. N. (2015). Research Domain Criteria: Toward future psychiatric nosologies. Dialogues in Clinical Neuroscience, 17(1), 89-97.

Dunne, M. N. (2008). Visual and verbal thinking [D. Phil. thesis]. University of Oxford.

Eigsti, I.-M., de Marchena, A. B., Schuh, J. M., \& Kelley, E. (2011). Language acquisition in autism spectrum disorders: A developmental review. Research in Autism Spectrum Disorders, 5(2), 681-691. https://doi.org/10.1016/j.rasd.2010.09.001

Feest, U. (2012). Introspection as a method and introspection as a feature of consciousness. Inquiry, 55(1), 1-16. https://doi.org/10. 1080/0020174X.2012.643619

Gale, C. M., Eikeseth, S., \& Klintwall, L. (2019). Children with autism show atypical preference for non-social stimuli. Scientific Reports, 9(1), 10355. https://doi.org/10.1038/s41598-019-46705-8

Gil, S., Chambres, P., Hyvert, C., Fanget, M., \& Droit-Volet, S. (2012). Children with autism spectrum disorders have "the working raw material" for time perception. PLoS ONE, 7(11), e49116. https:// doi.org/10.1371/journal.pone.0049116

Grandin, T. (2009). How does visual thinking work in the mind of a person with autism? A personal account. Philosophical Transactions of the Royal Society b: Biological Sciences, 364(1522), 1437-1442. https://doi.org/10.1098/rstb.2008.0297

Happé, F., \& Frith, U. (2020a). Annual research review: Looking back to look forward-Changes in the concept of autism and implications for future research. Journal of Child Psychology and Psychiatry, 61(3), 218-232. https://doi.org/10.1111/jcpp.13176

Happé, F., \& Frith, U. (2020b). Dimensional or categorical approaches to autism? Both are needed. A reply to Nick Chown and Julia Leatherland. Journal of Autism and Developmental Disorders. https://doi.org/10.1007/s10803-020-04728-5

Harris, P. L., \& Leevers, H. J. (2000). Pretending, imagery and selfawareness in autism. In Understanding other minds: Perspectives from developmental cognitive neuroscience, 2nd ed (pp. 182-202). Oxford University Press. 
Hoekstra, R. A., Bartels, M., Verweij, C. J. H., \& Boomsma, D. I. (2007). Heritability of autistic traits in the general population. Archives of Pediatrics \& Adolescent Medicine, 161(4), 372. https://doi.org/10.1001/archpedi.161.4.372

Hurlburt, R. T., Happé, F., \& Frith, U. (1994). Sampling the form of inner experience in three adults with Asperger syndrome. Psychological Medicine, 24(2), 385-395. https://doi.org/10.1017/S0033 291700027367

Ingersoll, B., \& Wainer, A. (2014). The broader autism phenotype. In F. R. Volkmar, S. J. Rogers, R. Paul, \& K. A. Pelphrey (Eds.), Handbook of autism and pervasive developmental disorders (Vol. 1, pp. 28-56). John Wiley \& Sons Inc.

Jivraj, J., Sacrey, L.-A., Newton, A., Nicholas, D., \& Zwaigenbaum, L. (2014). Assessing the influence of researcher-partner involvement on the process and outcomes of participatory research in autism spectrum disorder and neurodevelopmental disorders: A scoping review. Autism, 18(7), 782-793. https:// doi.org/10.1177/1362361314539858

Jobe, L. E., \& Williams White, S. (2007). Loneliness, social relationships, and a broader autism phenotype in college students. Personality and Individual Differences, 42(8), 1479-1489. https:// doi.org/10.1016/j.paid.2006.10.021

Jurek, L., Longuet, Y., Baltazar, M., Amestoy, A., Schmitt, V., Desmurget, M., \& Geoffray, M.-M. (2019). How did I get so late so soon? A review of time processing and management in autism. Behavioural Brain Research, 374, 112121. https://doi.org/10. 1016/j.bbr.2019.112121

Kamio, Y., \& Toichi, M. (2000). Dual access to semantics in autism: Is pictorial access superior to verbal access? Journal of Child Psychology and Psychiatry, 41(7), 859-867. https://doi.org/10. 1111/1469-7610.00673

Kunda, M., \& Goel, A. K. (2011). Thinking in pictures as a cognitive account of autism. Journal of Autism and Developmental Disorders, 41(9), 1157-1177. https://doi.org/10.1007/ s10803-010-1137-1

Kwok, E. Y. L., Brown, H. M., Smyth, R. E., \& Oram Cardy, J. (2015). Meta-analysis of receptive and expressive language skills in autism spectrum disorder. Research in Autism Spectrum Disorders, 9, 202-222. https://doi.org/10.1016/j.rasd. 2014.10.008

Lawson, W. (2001). Understanding and working with the spectrum of autism: An insider's view. Jessica Kingsley Publishers.

Loomes, R., Hull, L., \& Mandy, W. P. L. (2017). What is the male-tofemale ratio in autism spectrum disorder? A systematic review and meta-analysis. Journal of the American Academy of Child \& Adolescent Psychiatry, 56(6), 466-474. https://doi.org/10. 1016/j.jaac.2017.03.013

Lundin, A., Kosidou, K., \& Dalman, C. (2019). Measuring autism traits in the adult general population with the Brief AutismSpectrum Quotient, AQ-10: Findings from the Stockholm public health cohort. Journal of Autism and Developmental Disorders, 49(2), 773-780. https://doi.org/10.1007/s10803-018-3749-9

McGregor, E., Whiten, A., \& Blackburn, P. (1998a). Teaching theory of mind by highlighting intention and illustrating thoughts: A comparison of their effectiveness with 3 -year-olds and autistic individuals. British Journal of Developmental Psychology, 16(3), 281-300. https://doi.org/10.1111/j.2044-835X.1998. tb00753.x

McGregor, E., Whiten, A., \& Blackburn, P. (1998b). Transfer of the picture-in-the-head analogy to natural contexts to aid false belief understanding in autism. Autism, 2(4), 367-387. https:// doi.org/10.1177/1362361398024004

Montangero, J. (1996). Understanding changes in time. Taylor \& Francis.

Montangero, J., \& Pons, F. (1995). L'introduction du passé et du futur dans la description d'une situation présente: Étude du développement de la tendance diachronique. L'année Psychologique, 95, 621-644.

Montangero, J., Pons, F., \& Cattin, J.-P. (2000). The diachronic approach and solutions to interpersonal conflicts. British Journal of Developmental Psychology, 18(3), 415-429. https://doi. org/10.1348/026151000165779

Moore, B. D., Brooks, P. J., \& Rabin, L. A. (2014). Comparison of diachronic thinking and event ordering in 5- to 10-year-old children. International Journal of Behavioral Development, 38(3), 282-292. https://doi.org/10.1177/0165025414520806

Nayar, K., Sealock, J. M., Maltman, N., Bush, L., Cook, E. H., Davis, L. K., \& Losh, M. (2021). Elevated polygenic burden for autism spectrum disorder is associated with the broad autism phenotype in mothers of individuals with autism spectrum disorder. Biological Psychiatry, 89(5), 476-485. https://doi.org/10. 1016/j.biopsych.2020.08.029

Nicolaidis, C., Raymaker, D., Kapp, S. K., Baggs, A., Ashkenazy, E., McDonald, K., Weiner, M., Maslak, J., Hunter, M., \& Joyce, A. (2019). The AASPIRE practice-based guidelines for the inclusion of autistic adults in research as co-researchers and study participants. Autism, 23(8), 2007-2019. https://doi.org/10.1177/ 1362361319830523

Overweg, J., Hartman, C. A., \& Hendriks, P. (2018). Temporarily out of order: Temporal perspective taking in language in children with autism spectrum disorder. Frontiers in Psychology, 9. https://doi.org/10.3389/fpsyg.2018.01663

Paynter, J., \& Peterson, C. C. (2013). Further evidence of benefits of thought-bubble training for theory of mind development in children with autism spectrum disorders. Research in Autism Spectrum Disorders, 7(2), 344-348. https://doi.org/10.1016/j. rasd.2012.10.001

Pons, F., Harris, P. L., \& de Rosnay, M. (2012). Piaget and consciousness: Retrospect and prospect. In E. Martí \& C. Rodríguez (Eds.), After Piaget (pp. 95-122). Routledge.

Pons, F., \& Montangero, J. (1999). Is the diachronic thought a specific reasoning ability? Swiss Journal of Psychology, 58(3), 191-200. https://doi.org/10.1024/1421-0185.58.3.191

Pons, F., Montangero, J., Quadir, N., \& Bazan, C. (2002). Tendencia diacrónica y niveles de conocimiento en le niño: El caso de la comprensión de la enfermedad. Psicologia Iberoamericana, $10,23-30$

Poole, D., Gowen, E., Poliakoff, E., \& Jones, L. A. (2021). 'No idea of time': Parents report differences in autistic children's behaviour relating to time in a mixed-methods study. Autism, 13623613211010014. https://doi.org/10.1177/1362361321 1010014

Soulières, I., Zeffiro, T. A., Girard, M. L., \& Mottron, L. (2011). Enhanced mental image mapping in autism. Neuropsychologia, 49(5), 848-857. https://doi.org/10.1016/j.neuropsychologia. 2011.01.027

Swettenham, J. G. (1996). What's inside someone's head? Conceiving of the mind as a camera helps children with autism acquire an alternative to a theory of mind. Cognitive Neuropsychiatry, 1(1), 73-88. https://doi.org/10.1080/135468096396712

Velikonja, T., Fett, A.-K., \& Velthorst, E. (2019). Patterns of nonsocial and social cognitive functioning in adults with autism spectrum disorder: A systematic review and meta-analysis. JAMA Psychiatry, 76(2), 135-151. https://doi.org/10.1001/jamapsychi atry.2018.3645

Vogel, D., Falter-Wagner, C. M., Schoofs, T., Krämer, K., Kupke, C., \& Vogeley, K. (2019). Interrupted time experience in autism spectrum disorder: Empirical evidence from content analysis. Journal of Autism and Developmental Disorders, 49(1), 22-33. https://doi.org/10.1007/s10803-018-3771-y

Wellman, H. M., Baron-Cohen, S., Caswell, R., Gomez, J. C., Swettenham, J., Toye, E., \& Lagattuta, K. (2002). 
Thought-bubbles help children with autism acquire an alternative to a theory of mind. Autism, 6(4), 343-363. https://doi.org/ $10.1177 / 1362361302006004003$

Whitehouse, A. J. O., Maybery, M. T., \& Durkin, K. (2006). Inner speech impairments in autism. Journal of Child Psychology and Psychiatry, 47(8), 857-865. https://doi.org/10.1111/j.14697610.2006.01624.x

Wigham, S., Rodgers, J., Berney, T., Le Couteur, A., Ingham, B., \& Parr, J. R. (2019). Psychometric properties of questionnaires and diagnostic measures for autism spectrum disorders in adults: A systematic review. Autism, 23(2), 287-305. https:// doi.org/10.1177/1362361317748245

Williams, D. (1996). Autism-An inside-out approach: An innovative look at the mechanics of 'autism' and its developmental 'cousins'. Jessica Kingsley Publishers.
Williams, D. M., Bowler, D. M., \& Jarrold, C. (2012). Inner speech is used to mediate short-term memory, but not planning, among intellectually high-functioning adults with autism spectrum disorder. Development and Psychopathology, 24(1), 225-239. https:// doi.org/10.1017/S0954579411000794

Williams, D. M., Peng, C., \& Wallace, G. L. (2016). Verbal thinking and inner speech use in autism spectrum disorder. $\mathrm{Neu}$ ropsychology Review, 26(4), 394-419. https://doi.org/10.1007/ s11065-016-9328-y

Publisher's Note Springer Nature remains neutral with regard to jurisdictional claims in published maps and institutional affiliations. 